\title{
Colestasis neonatal: reporte de 21 casos en un hospital infantil de Cartagena, Colombia
}

\section{Report of 21 Cases of Neonatal Cholestasis in a Children's Hospital in Cartagena, Colombia}

\author{
Jorge José Mendoza H, MD, ${ }^{1}$ Rodrigo De Vivero C, MD, ${ }^{2}$ Nelson Muñoz A, MD, ${ }^{3}$ Amileth Suarez C, PhD, ${ }^{4}$ Carlos Moneriz P, PhD, ${ }^{5}$
}

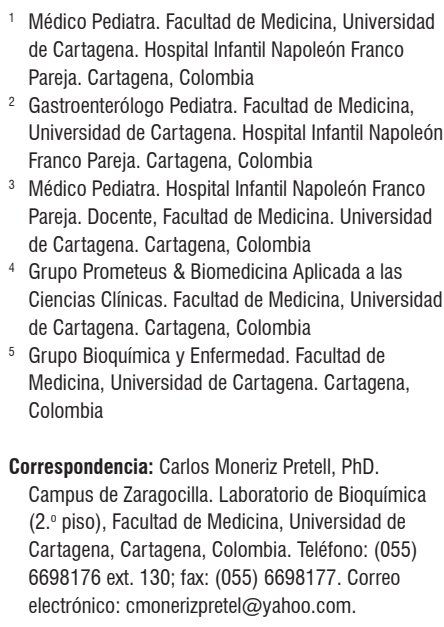

Médico Pediatra. Facultad de Medicina, Universidad de Cartagena. Hospital Infantil Napoleón Franco Pareja. Cartagena, Colombia

2 Gastroenterólogo Pediatra. Facultad de Medicina, Universidad de Cartagena. Hospital Infantil Napoleón Franco Pareja. Cartagena, Colombia

3 Médico Pediatra. Hospital Infantil Napoleón Franco Pareja. Docente, Facultad de Medicina. Universidad de Cartagena. Cartagena, Colombia

Grupo Prometeus \& Biomedicina Aplicada a las Ciencias Clínicas. Facultad de Medicina, Universidad de Cartagena. Cartagena, Colombia

${ }^{5}$ Grupo Bioquímica y Enfermedad. Facultad de Medicina, Universidad de Cartagena. Cartagena, Colombia

Correspondencia: Carlos Moneriz Pretell, $\mathrm{PhD}$. Campus de Zaragocilla. Laboratorio de Bioquímica (2. ${ }^{\circ}$ piso), Facultad de Medicina, Universidad de Cartagena, Cartagena, Colombia. Teléfono: (055) 6698176 ext. 130; fax: (055) 6698177. Correo electrónico: cmonerizpretel@yahoo.com.

Financiación: Vicerrectoría de Investigaciones de la Universidad de Cartagena en el plan de fortalecimiento y sostenibilidad del grupo Bioquímica y Enfermedad, reconocido por Colciencias.

\begin{abstract}
Resumen
La colestasis es una alteración en el flujo biliar que se presenta por la disminución o el cese de excreción biliar. En la actualidad, son pocos los estudios en Colombia sobre esta patología. Se presentan 21 casos de colestasis neonatal en un hospital infantil de la ciudad de Cartagena (Colombia) entre 2010 y 2013, con el objetivo de caracterizar la etiología y clínica de la enfermedad. Se seleccionaron los pacientes entre 0 y 3 meses de edad con bilirrubina directa >2 mg/dL. En este estudio se encontró que según el género, el 52,4\% fueron de sexo masculino y el $47,6 \%$, de sexo femenino. La edad gestacional predominante fue a término en el $76,2 \%$ y sin antecedentes perinatales en el $57,1 \%$. Los hallazgos clínicos se presentaron en los primeros 30 días de nacido en un $71 \%$ y 4 pacientes fueron remitidos a trasplante hepático. La etiología más frecuente fue de tipo infeccioso en 13 de los pacientes estudiados y 4 pacientes se relacionaron con atresias. La causa más frecuente de colestasis neonatal en este estudio resultó estar asociada con etiologías infecciosas. Sin embargo, las alteraciones obstructivas, como la atresia de vías biliares, siguen ocupando un renglón importante y requieren un estudio y manejo prioritario, dado su mejor pronóstico relacionado con la intervención temprana.
\end{abstract}

\section{Palabras claves}

Colestasis neonatal, bilirrubinas, atresia de vías biliares, ictericia neonatal, sepsis neonatal.

\section{Abstract}

Cholestasis is an alteration in the flow of bile resulting from decreases or cessation of biliary excretion. To date, there have been only a few studies on this topic in Colombia. This article presents twenty-one cases of neonatal cholestasis from a Children's Hospital in Cartagena, Colombia that occurred between 2010 and 2013. The aim of this study is to characterize the etiology and clinical characteristics of the disease. Patients between birth and 3 months old with direct bilirubin levels over $2 \mathrm{mg} / \mathrm{dl}$ were selected. By gender, $52.4 \%$ of the patients were male, and $47.6 \%$ were female. $76.2 \%$ of the patients were full term, and $57.1 \%$ had no perinatal antecedents. Clinical symptoms presented in the first 30 days after birth in $71 \%$ of the patients, and 4 patients were referred for liver transplantation. The most common etiology was infectious (13 patients), and 4 patients had atresia. The most common cause of neonatal cholestasis in this study was infection, but obstructive disorders such as biliary atresia still account for an important proportion of the patients. They require priority study and handling because early intervention results in better prognoses.

\section{Keywords}

Neonatal cholestasis, bilirubin, biliary atresia, neonatal jaundice, neonatal sepsis. 


\section{INTRODUCCIÓN}

La colestasis es un síndrome de disfunción hepática con alteraciones en el flujo biliar, definido analíticamente por la elevación de los ácidos biliares séricos y de la bilirrubina directa $(>2 \mathrm{mg} / \mathrm{dL})(1)$ o cuando la bilirrubina directa corresponde a más del $20 \%$ de la bilirrubina total. Se produce por una obstrucción del flujo a través del árbol biliar intra o extrahepático, que ocasiona un acumulo de substancias que se excretan normalmente en la bilis. Suele presen- tarse con ictericia prolongada, orina oscura, heces pálidas, hepatomegalia y prurito dentro de las primeras 4 semanas de vida $(2,3)$. El término colestasis neonatal involucra el período del recién nacido hasta los 3 meses de edad $(1,4)$.

Los ácidos biliares son tóxicos para las membranas y deben ser transportados de forma protegida, lo que se logra mediante la acción de diversas proteínas y transporte vesicular en el interior del hepatocito (figura 1) (5). En un hepatocito colestásico se altera la funcionalidad de las proteínas trasportadoras y se afecta el transporte vesicular por

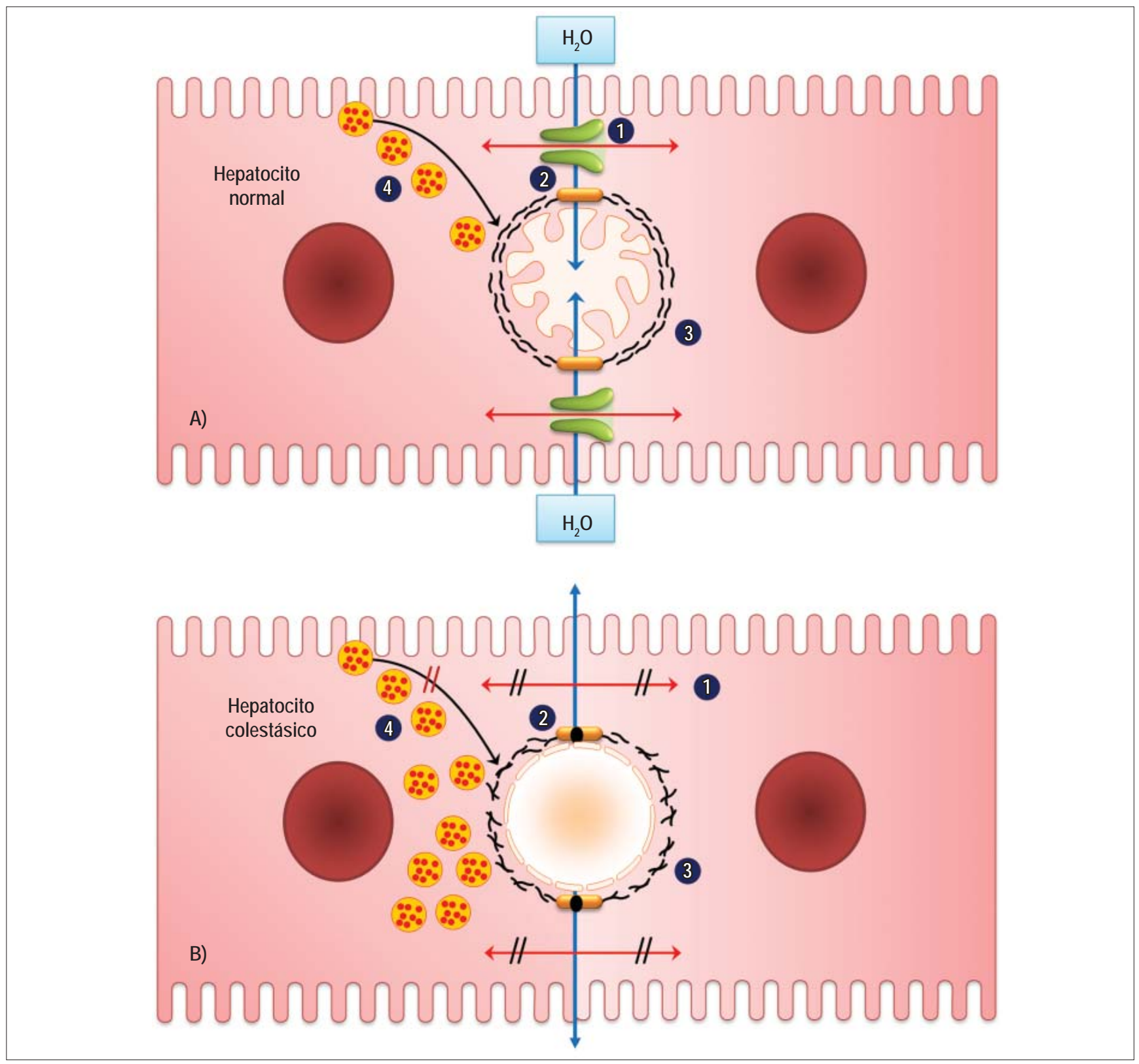

Figura 1. Transporte biliar en el hepatocito. A. Hepatocito normal: 1) proteína de unión; 2) unión estrecha; 3) red de actina-miosina; 4) vía vesicular transcitótica. B. Hepatocito con colestasis: 1) pérdida de uniones; 2) uniones con fugas; 3) desarreglo actina-miosina; 4) interrupción de la vía vesicular. Fuente: propia de autores. 
la desorganización de los microtúbulos de la célula, lo que ocasiona acumulación de los componentes biliares $(6,7)$.

Este síndrome o alteración clínica se presenta en 1 de cada 2500 o 5000 nacidos vivos (8). Sin embargo, esta tasa por individuo puede variar según la raza y el sexo; por ejemplo, la incidencia en Europa es de 1 por cada 18000 $(1,9,10)$.

La incidencia de la colestasis neonatal a nivel mundial es baja, lo cual hace que se subestime la patología. Por otra parte, cabe resaltar que la etiología de dicha alteración es multifactorial, por lo que pueden estar involucrados factores genéticos, ambientales y hormonales $(11,12)$. Puede estar relacionada con hepatitis neonatal, obstrucción biliar, síndromes colestásicos genéticos y enfermedades metabólicas (13). Con base en lo anterior, es importante que todos los neonatos que presenten síntomas como ictericia prolongada sean estudiados y mediante un diagnóstico acertado y tratamiento exitoso se pueda garantizar su supervivencia (14-16). Algunos autores afirman que no siempre es posible identificar las causas de la colestasis, siendo necesario y urgente para poder tratar al paciente (17). La atresia de la vía biliar y la hepatitis neonatal comprenden del $55 \%$ al $65 \%$ de todos los casos de colestasis neonatal $(1,4)$. En cuanto al tratamiento, puede ser médico o qui- rúrgico, dependiendo del caso, y algunas veces se requiere trasplante hepático, que es la terapia estandarizada para la insuficiencia hepática aguda y crónica; más del 50\% de los trasplantes se deben a enfermedades colestásicas (18).

El hígado de los neonatos es inmaduro (19) y presenta una desventaja frente al de los niños con más edad, ya que posee menos concentración de ácidos biliares y la expresión de los transportadores hepatobiliares está disminuida (20). Por tanto, esta condición predispone al recién nacido a hemorragias, encefalopatías, déficit de vitaminas A, D, K y E, aparición de ictericia, entre otros $(4,21)$.

La colestasis neonatal es un problema a nivel mundial, con una mortalidad estimada en un 15\%-50\%. Se encuentra asociada con factores perinatales que en un momento determinado pueden ayudar a realizar un diagnóstico adecuado, lo que llevaría a un manejo óptimo que se reflejaría en la disminución de las consecuencias graves como sepsis, insuficiencia hepática y muerte $(8,22)$.

En casos de sospecha de colestasis, el dato principal y más importante en la evaluación es el color de las deposiciones. Si las deposiciones son acólicas, la atresia biliar debe ser diagnosticada o excluida (figura 2). Si se excluye una patología obstructiva del árbol biliar extrahepático, el diagnóstico de atresia biliar es probable. Deben efectuarse

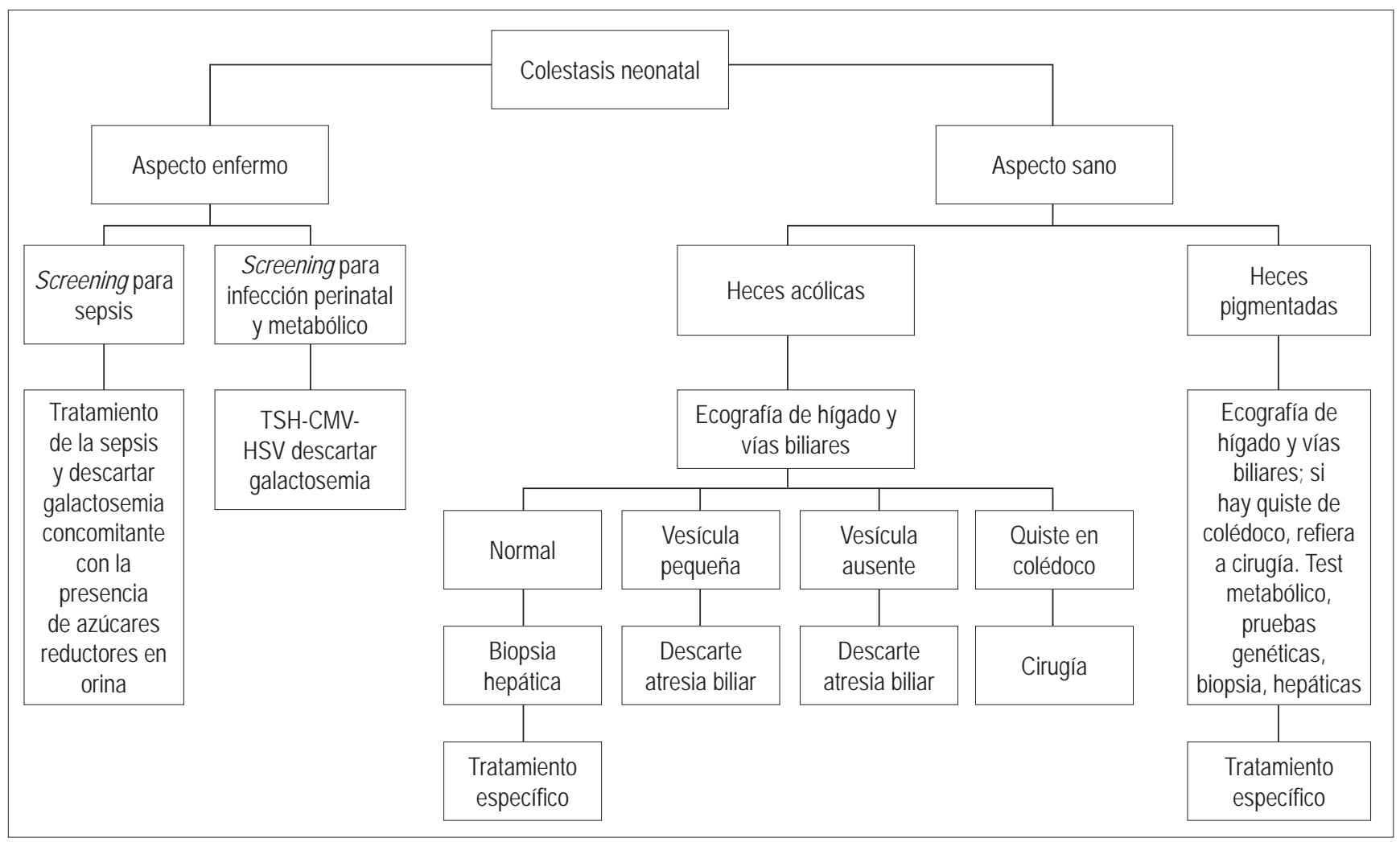

Figura 2. Algoritmo diagnóstico y manejo de la colestasis neonatal. 
una colangiografía y una biopsia hepática. Si la vía biliar no es permeable, debe practicarse una intervención de Kasai. Por el contrario, si la vía biliar es permeable, debe considerarse otro diagnóstico y una posible colangitis esclerosante neonatal (12).

El propósito del presente artículo es identificar las principales características etiológicas y clínicas de los pacientes con colestasis neonatal en el Hospital Infantil Napoleón Franco Pareja de la ciudad de Cartagena, Colombia. Es importante resaltar que en el territorio colombiano son pocos los estudios sobre esta enfermedad. Por tanto, esta investigación está dirigida a proponer nuevo conocimiento, determinar elementos de apoyo en el diagnóstico más preciso y mejorar la atención neonatal.

\section{PRESENTACIÓN DE LOS CASOS}

Se presentan 21 pacientes con colestasis neonatal, entre 0 y 3 meses de edad, con bilirrubina directa $>2 \mathrm{mg} / \mathrm{dL}$, que fueron internados en las salas de hospitalización general del Hospital Infantil Napoleón Franco Pareja de la ciudad de Cartagena (Colombia), durante el período comprendido entre enero de 2010 y abril de 2013. Los datos fueron recogidos a partir de la realización de entrevistas a los familiares responsables de los pacientes y al personal médico responsable de su cuidado, así como de la revisión de las historias clínicas. Los parámetros que se evaluaron fueron: edad, sexo, manifestaciones clínicas (tabla 1), antecedentes perinatales, resultados de laboratorios como bilirrubinas, TORCH, entre otros (tabla 2) y etiologías relacionadas. Este estudio se clasificó en la categoría de investigación sin riesgo, según el artículo 11 de la resolución del Ministerio de Salud No. 008430 de 1993.

El grupo de estudio estuvo integrado por una proporción muy similar en cuanto al género. La edad gestacional más frecuente fue "a término", con un 76\% (16 pacientes), mientras que los pacientes pretérmino correspondieron a un $24 \%$ ( 5 pacientes) (tabla 1). Los hallazgos clínicos más frecuentes fueron la presencia de ictericia y hepatomegalia, aunque en algunos casos no hubo acolia ni coluria. Los hallazgos clínicos se presentaron con mayor frecuencia al mes de vida, con promedio de edad al momento de la aparición clínica de 33,6 624 días.

En el estudio se observó que una proporción importante no tenía antecedentes perinatales de ningún tipo $(57,1 \%)$ y 4 pacientes presentaron antecedentes de sepsis neonatal

Tabla 1. Características básicas y clínicas de los nacidos vivos con presencia de colestasis neonatal en el Hospital Infantil Napoleón Franco Pareja (2010-2013).

\begin{tabular}{|c|c|c|c|c|c|c|c|c|c|c|}
\hline Paciente & Sexo & $\begin{array}{c}\text { Edad } \\
\text { gestacional }\end{array}$ & Parto & Ictericia & Hepatomegalia & Esplenomegalia & Acolia & Coluria & Fiebre & $\begin{array}{c}\text { Edad (días) } \\
\text { hallazgos }\end{array}$ \\
\hline n. ${ }^{0} 1$ & $\mathrm{~F}$ & Término & Cesárea & + & + & + & + & - & - & 60 \\
\hline n. ${ }^{2} 2$ & $\mathrm{~F}$ & Término & Cesárea & + & + & + & + & + & - & 1 \\
\hline n. 3 & $\mathrm{~F}$ & Término & Cesárea & + & + & + & + & + & - & 7 \\
\hline n. ${ }^{\circ} 4$ & M & Término & Cesárea & + & - & - & + & - & - & 30 \\
\hline n. ${ }^{\circ} 5$ & M & Término & Cesárea & + & - & - & - & - & - & 60 \\
\hline ก. ${ }^{\circ} 6$ & $\mathrm{~F}$ & Término & Vaginal & + & - & - & + & - & - & 30 \\
\hline n. ${ }^{0} 7$ & M & Término & Cesárea & + & + & - & - & - & - & 60 \\
\hline n. ${ }^{\circ} 8$ & $\mathrm{~F}$ & Término & Cesárea & + & + & - & - & + & - & 30 \\
\hline ก. 99 & M & Término & Vaginal & + & + & + & + & + & - & 90 \\
\hline n. ${ }^{\circ} 10$ & $\mathrm{~F}$ & Término & Vaginal & + & + & - & - & - & + & 30 \\
\hline n. ${ }^{\circ} 11$ & M & Término & Vaginal & + & - & - & - & - & + & 30 \\
\hline n. ${ }^{\circ} 12$ & M & Término & Vaginal & + & - & - & - & - & + & 30 \\
\hline n. ${ }^{\circ} 13$ & M & Término & Vaginal & + & - & - & - & - & - & 30 \\
\hline n. ${ }^{\circ} 14$ & M & Pretérmino & Cesárea & + & - & - & - & + & - & 2 \\
\hline n. ${ }^{0} 15$ & M & Término & Cesárea & + & - & - & - & + & - & 4 \\
\hline n. ${ }^{\circ} 16$ & $\mathrm{~F}$ & Pretérmino & Cesárea & + & - & - & - & - & - & 60 \\
\hline n. ${ }^{\circ} 17$ & M & Término & Cesárea & + & - & - & - & - & - & 1 \\
\hline n. ${ }^{\circ} 18$ & M & Término & Vaginal & + & + & + & + & + & - & 30 \\
\hline ก. 19 & $\mathrm{~F}$ & Pretérmino & Cesárea & + & + & + & + & + & + & 30 \\
\hline n. ${ }^{0} 20$ & $\mathrm{~F}$ & Pretérmino & Cesárea & + & + & + & - & - & - & 30 \\
\hline n. ${ }^{2} 21$ & $\mathrm{~F}$ & Pretérmino & Vaginal & + & - & - & - & - & - & 60 \\
\hline
\end{tabular}


(14,3\%), de la siguiente forma (tabla 2): 1 paciente presentó sepsis neonatal temprana (paciente n. $^{\circ} 3$ ); 2 pacientes presentaron sepsis neonatal asociada con nutrición parenteral (pacientes n. ${ }^{\circ} 19$ y n. ${ }^{\circ} 20$ ) y 1 paciente con sepsis neonatal estuvo asociado con atresia intestinal y ano imperforado (paciente n. ${ }^{\circ}$ 21). Los 5 pacientes restantes presentaron antecedentes de ictericia neonatal ( . $^{\circ}$ 9), enterocolitis necrosante (.$\left.^{\circ} 17\right)$, megacolon congénito (n..$\left.^{\circ} 15\right)$, hepatitis neonatal (n. $\left.{ }^{\circ} 8\right)$ y múltiples antecedentes (n. $\left.{ }^{\circ} 14\right)$ : aloimnuización severa, síndrome de Down, atresia duodenal, trombocitopenia, choque séptico, conducto arterioso persistente.

Por otra parte, los niveles de bilirrubina directa más frecuentemente observadosfueronalrededorde $4 \mathrm{mg} / \mathrm{dLy}$ solo el paciente n. $^{\circ} 13$ presentó un valor de $21 \mathrm{mg} / \mathrm{dL}$ (tabla 2 ). El promedio de bilirrubina directa de todos los pacientes fue de 7,2 $\pm 4,6 \mathrm{mg} / \mathrm{dL}$. El perfil hepático de todos los pacientes mostró alteración, que cursó con transaminasas y tiempos de coagulación elevados. Además, la mayoría de los pacientes presentaron disminución de la hemoglobina y del hematocrito.

Con respecto a la etiología, se encontraron 13 pacientes asociados con causa infecciosa, 4 del total de nacidos vivos estudiados presentaron atresia en vías biliares, 3 pacientes con causa idiopática y solo 1 paciente presentó un quiste en el colédoco (figura 3). Cabe resaltar que el nivel de bilirrubina directa de $21 \mathrm{mg} / \mathrm{dL}$ más alto estuvo asociado con infección neonatal (toxoplasmosis) y no con atresia de vías biliares. También se observó que dentro de las principales causas infecciosas se encontraron 8 pacientes asociados con sepsis neonatal (62\%), 4 pacientes positivos para infección con citomegalovirus (CMV) (31\%) y 1 paciente con toxoplasmosis (8\%).

Los lactantes que fueron remitidos a trasplantes se caracterizaron por la edad gestacional a término, presencia de antecedentes perinatales, acolia, coluria, aumento de AST y ALT, bilirrubinas directas de 4 a $16,6 \mathrm{mg} / \mathrm{dL}$ y tiempos de coagulación prolongados. Por último, es de resaltar que el $75 \%$ de los pacientes del grupo de atresias (figura 4) fue remitido a trasplantes frente a un $8 \%$ del grupo de causa infecciosa.

\section{DISCUSIÓN}

A nivel mundial, la incidencia de colestasis es muy baja y puede variar según la raza y el género (23). En este estudio se diagnosticaron 21 pacientes con colestasis neonatal

Tabla 2. Antecedentes perinatales, pruebas de laboratorio y etiología relacionada de los nacidos vivos con presencia de colestasis neonatal en el Hospital Infantil Napoleón Franco Pareja (2010-2013).

\begin{tabular}{|c|c|c|c|c|c|c|}
\hline Paciente & Antecedentes perinatales & BT & $\mathrm{BD}$ & Toxoplasma & CMV & Etiología \\
\hline n. ${ }^{\circ} 1$ & - & 11,53 & 6,01 & - & - & Atresia \\
\hline n. ${ }^{2}$ & - & 8,7 & 5 & - & - & Atresia \\
\hline n. ${ }^{\circ} 3$ & Sepsis neonatal temprana & 7,6 & 4 & - & - & Atresia \\
\hline n. ${ }^{\circ} 4$ & - & 7,95 & 4 & - & - & Idiopática \\
\hline n. ${ }^{5}$ & - & 10,4 & 5,5 & - & - & Idiopática \\
\hline n. ${ }^{\circ} 6$ & - & 25,2 & 10,5 & - & + & Infección por CMV \\
\hline n. ${ }^{\circ} 7$ & - & 19,2 & 7,4 & - & + & Infección por CMV \\
\hline n. 8 & Hepatitis A neonatal & 28,6 & 16,6 & - & + & Infección por CMV \\
\hline n. ${ }^{9} 9$ & Ictericia neonatal & 7,8 & 4 & - & - & Atresia \\
\hline n. ${ }^{\circ} 10$ & - & 14,8 & 8,1 & - & - & Sepsis neonatal \\
\hline n. ${ }^{\circ} 11$ & - & 5,6 & 2,4 & - & - & Sepsis neonatal \\
\hline n. ${ }^{1} 12$ & - & 13,2 & 6,4 & - & - & Sepsis neonatal \\
\hline n. ${ }^{\circ} 13$ & - & 25 & 21 & + & - & Toxoplasmosis \\
\hline n. ${ }^{\circ} 14$ & Múltiples & 29,6 & 12,4 & - & - & Sepsis neonatal \\
\hline n. ${ }^{1} 15$ & Megacolon congénito & 7,6 & 4,2 & - & - & Sepsis neonatal \\
\hline n. ${ }^{1} 16$ & - & 8,2 & 5,1 & - & - & Idiopática \\
\hline n. ${ }^{\circ} 17$ & Enterocolitis necrosante & 6,4 & 2,8 & - & - & Sepsis neonatal \\
\hline n. ${ }^{1} 18$ & - & 7,8 & 4 & - & - & Quiste colédoco \\
\hline n. ${ }^{\circ} 19$ & Sepsis neonatal asociada con NPT & 16,4 & 7,2 & - & - & Sepsis neonatal \\
\hline n. ${ }^{\circ} 20$ & Sepsis neonatal asociada con NPT & 16,4 & 7,2 & - & + & Infección por CMG \\
\hline n. ${ }^{2} 21$ & Ano imperforado & 21,8 & 7,6 & - & - & Sepsis neonatal \\
\hline
\end{tabular}

NPT: nutrición parenteral; CMV: citomegalovirus. 
durante un período de 3 años. Estos datos concuerdan con la incidencia estimada en la población de recién nacidos de la ciudad de Cartagena, Colombia, partiendo del hecho que el hospital infantil en estudio es el principal de la zona por tratarse del centro de referencia pediátrica.

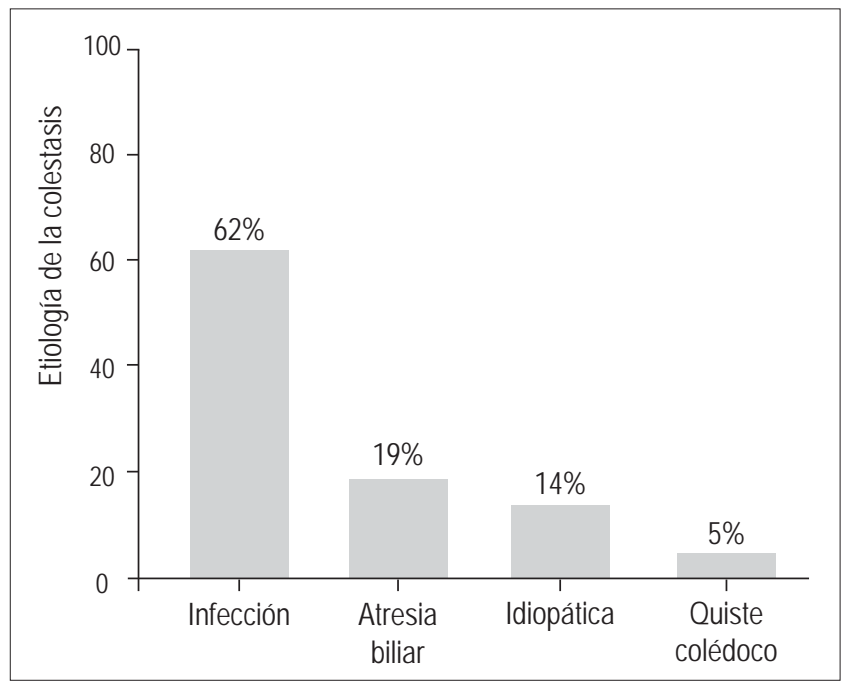

Figura 3. Distribución por etiologías de los nacidos vivos con presencia de colestasis neonatal en el Hospital Infantil Napoleón Franco Pareja (2010-2013).

En este estudio no se observaron diferencias significativas en cuanto al género, aunque sí se encontró una predominancia de edad gestacional a término. Estos datos resultaron ser similares a un estudio realizado por Álvarez y colaboradores en el Hospital Pediátrico William Soler de Cuba (9). Aunque en otro estudio retrospectivo realizado por Jacquemin y colaboradores (24) se encontró una predominancia de género masculino en los pacientes con colestasis neonatal. Por otra parte, los antecedentes perinatales fueron negativos en la mayoría de los casos; sin embargo, aquellos que evidenciaron antecedente desarrollaron síntomas y diagnósticos que incluso llevaron a remisión de trasplante.

Los resultados obtenidos de este estudio demuestran la fuerte asociación que tienen algunas fuentes etiológicas con la patología, como es la presencia de sepsis neonatal, así como también las atresias biliares. Estos resultados evidencian diferencias etiológicas con respecto a otros estudios realizados en otras zonas del mundo. Por ejemplo, un estudio llevado a cabo por Méndez y colaboradores (25), en Montevideo, evidenció que las principales etiologías encontradas fueron hepatitis (16 pacientes) y atresia (13 pacientes).

Las causas de colestasis por infecciones están asociadas con CMV, toxoplasmosis, rubéola, sífilis, herpes, sepsis bacteriana, entre otras (26-28); esta información coincide con el resultado del presente estudio. Las infecciones congénitas suelen compartir similitudes clínicas, como agrandamiento del hígado y del bazo, ictericia, neumonitis y una tendencia a la prematuridad o al crecimiento intrauterino deficiente (27). La presentación clínica con insuficiencia hepática neonatal es posible con cualquiera de estos agentes; no obstante, aunque se reporta que es más común con la infección por herpes simple, en este estudio se evidenció una mayor frecuencia en la infección por CMV.

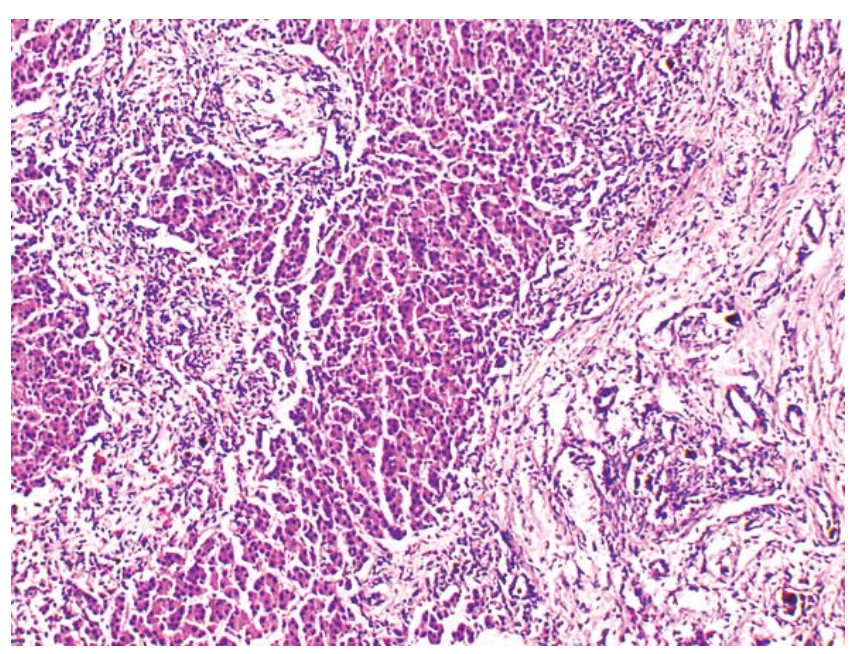

Figura 4. Biopsia hepática de un paciente (n. $\left.{ }^{\circ} 1\right)$ con etiología de atresia biliar. Cirrosis establecida secundaria a enfermedad fibrosante necroinflamatoria de las vías biliares. Los cortes muestran parénquima hepático con alteración en su histoarquitectura, en la que se observa un patrón de lesión tipo obstructivo, con expansión de los espacios portales, tapones biliares en conductos, fibrosis periportal y en puentes, dilatación de los espacios sinusoidales, colestasis lobulillar y focos de eritropoyesis extramedular. No se observaron granulomas, inclusiones por microorganismos, transformación de células gigantes, ni malignidad en la muestra.

En esta investigación se encontraron 4 pacientes (19\% del total) con colestasis neonatal y asociados con infección por CMV. La presencia del CMV es la causa más común de infección congénita, que afecta al 1\%-2\% de los recién nacidos, la mayoría de los cuales son asintomáticos (27). Los hallazgos clínicos incluyen una erupción petequial, hepatoesplenomegalia e ictericia en el 60\%-80\% (27). La infección por CMV puede causar insuficiencia de hígado neonatal, y aunque la literatura lo reporta como poco común, en este estudio se encontró una proporción alta de pacientes con colestasis neonatal y asociados con este tipo de infección.

Los resultados en cuanto a la toxoplasmosis congénita, que se presentó en el 5\% de los pacientes, son una alerta para nuestra medio y coinciden con los casos reportados por otros investigadores $(29,30)$. Un estudio realizado en Brasil confirma que la toxoplasmosis en dicho país tiene 
una incidencia que varía de 2 a 20:10 000 nacidos. Dicho estudio de caso presenta bilirrubina directa de $35,6 \mathrm{mg} / \mathrm{dL}$ y concluye que la toxoplasmosis es una enfermedad de alta morbilidad que debe ser prevenida mediante tratamiento durante el período de vida (31).

Sin embargo, esto nos evidencia que opuesto a la literatura, la principal etiología o la más frecuente en este estudio fue la de carácter infeccioso. Resulta interesante saber que los resultados, a diferencia de la literatura, en donde se informa que las atresias de las vías biliares son las más frecuentes $(15,18,32)$, en nuestra población muestran mayores casos de sepsis e infección por CMV en comparación con los casos de atresias biliares. Este hallazgo nos hace pensar que la coincidencia de este tipo de etiología infecciosa presentado en los casos de colestasis neonatal sea un factor importante a estudiar en nuestra población.

Un estudio realizado en Medellín, Colombia, coincide con el presente estudio de acuerdo con la presencia de manifestaciones clínicas como ictericia, acolia y coluria (33). Sin embargo, la causa más frecuente fue la atresia (18 niños, 28\%) (33), lo que difiere de los resultados obtenidos en la presente investigación.

La atresia de vías biliares es la causa más frecuente de trasplante hepático pediátrico, dato que concuerda con los encontrados en este estudio. Sin embargo, pueden existir otras enfermedades hepáticas diferentes en su origen y evolución que requieran de este tratamiento (34). Hasta el $60 \%$ de los pacientes con atresia de vías biliares puede necesitar un trasplante en los primeros 10 años de vida; incluso aquellos en los que la cirugía consigue restablecer el flujo biliar pueden precisar de un trasplante en la segunda o tercera década (18).

En esta investigación se encontró solo 1 paciente con quiste en el colédoco. Esta alteración se refiere a un grupo de malformaciones congénitas en el sistema biliar. El más común es un tipo fusiforme, a veces en forma de salchicha, con dilatación de las vías biliares extrahepáticas. Los quistes de colédoco se identifican cada vez más en el feto por ecografía prenatal y han sido encontrados a las 15-16 semanas de gestación (27). El diagnóstico debe confirmarse inmediatamente después del nacimiento. La mayoría de estos niños tiene hiperbilirrubinemia conjugada, y la cirugía debe realizarse con prontitud.

Por último, la evaluación diagnóstica del recién nacido con colestasis debe llevarse a cabo de un modo sistematizado, lógico y costo-efectivo. Un equipo coordinado integrado por clínicos, cirujanos, radiólogos y bioquímicos debe minimizar la realización de pruebas diagnósticas innecesarias y lograr un diagnóstico correcto en el tiempo más corto posible.

\section{CONCLUSIÓN}

Con este reporte de casos se concluye que las características etiológicas de la colestasis no se encuentran precisas en la literatura, debido a que existe diversificación en cuanto a su clasificación. En algunas regiones la causa más frecuente puede ser la atresia de vías biliares y en otras zonas, la causa infecciosa, como es el caso de este estudio. Por lo tanto, los procesos diagnósticos para los neonatos deben comprender todos los estudios necesarios para así determinar la causa más prevalente y abordar el tratamiento de la manera más acertada, teniendo en cuenta el género, la raza y la localización geográfica.

\section{Agradecimientos}

A la Facultad de Medicina de la Universidad de Cartagena, al Hospital Infantil Napoleón Franco Pareja, que facilitó el acceso a la información y a la Doctora Katherine Redondo por su aporte en el análisis histopatólogico.

\section{Conflicto de intereses}

Los autores manifiestan que no presentan conflicto de interés con respecto a esta investigación.

\section{Financiación}

Vicerrectoría de Investigaciones de la Universidad de Cartagena, en el plan de fortalecimiento y sostenibilidad del grupo Bioquímica y Enfermedad, reconocido por Colciencias.

\section{REFERENCIAS}

1. Frauca E, Muñoz G. Conestasis en el lactante. En: Protocolos de gastroenterología, hepatología y nutrición (AEP). 2. ${ }^{a}$ edición. España: Ergón S.A. ed; 2010. p. 177-87.

2. Samyn M, Mieli-Vergani G. Liver and biliary disease in infancy. Medicine 2007;35(2):61-6.

3. Bellomo-Brandao MA, Porta G, Hessel G. Clinical and laboratory evaluation of 101 patients with intrahepatic neonatal cholestasis. Arq Gastroenterol 2008;45(2):152-5.

4. Heller S. Colestasis neonatal. MedUNAB 2005;8(1):S1825.

5. Wagner M, Zollner G, Trauner M. New molecular insights into the mechanisms of cholestasis. J Hepatol 2009;51(3):565-80.

6. Trauner M, Meier PJ, Boyer JL. Molecular pathogenesis of cholestasis. N Engl J Med. 1998 Oct 22;339(17):1217-27.

7. McKiernan PJ. Neonatal cholestasis. Semin Neonatol 2002; $7(2): 153-65$. 
8. De Bruyne R, Van Biervliet S, Vande Velde S, Van Winckel M. Clinical practice: neonatal cholestasis. Eur J Pediatr $2011 ; 170(3): 279-84$.

9. Hondal Alvarez N, Silverio García C. Comportamiento de la colestasis del recién nacido y del lactante en el Hospital Pediátrico "William Soler". Rev Cub Pediatr 2010;82:13-9.

10. Ciocca M, Alvarez F. Transient neonatal cholestasis. Arch Argent Pediatr 2011;109(2):163-6.

11. Lorente S, Montoro MA. Cholestasis of pregnancy. Gastroenterol Hepatol 2007;30(9):541-7.

12. Girard M, Lacaille F. Diagnosis of neonatal cholestasis. Annales Nestlé (English ed). 2008;66(3):109-20.

13. López Panqueva R. Aproximación al diagnóstico patológico de las enfermedades colestásicas. Rev Col Gastroenterol 2014;29:189-99.

14. Balistreri WF. Neonatal cholestasis: lessons from the past, issues for the future. Semin Liver Dis 1987;7(2):61-6.

15. Fischler B, Papadogiannakis N, Nemeth A. Aetiological factors in neonatal cholestasis. Acta Paediatr 2001;90(1):88-92.

16. Venigalla S, Gourley GR. Neonatal cholestasis. Semin Perinatol 2004;28(5):348-55.

17. Balistreri WF. Neonatal cholestasis. J Pediatr 1985;106(2):171-84.

18. Donat Aliaga E, Polo Miquel B, Ribes-Koninckx C. Atresia de vías biliares. An Pediatr (Barc) 2003;79(02):168-73.

19. Balistreri WF, Heubi JE, Suchy FJ. Immaturity of the enterohepatic circulation in early life: factors predisposing to "physiologic" maldigestion and cholestasis. J Pediatr Gastroenterol Nutr 1983;2(2):346-54.

20. Hierro L, Jara P. Childhood cholestasis and bile transporters. Gastroenterol Hepatol 2005;28(7):388-95.

21. López-Manzanares J, Medina Benítez E. Colestasis en el recién nacido y lactante. Orientación diagnóstica. An Pediatr 2003;58(02):162-7.

22. Moyer V, Freese DK, Whitington PF, Olson AD, Brewer F, Colletti RB, et al. Guideline for the evaluation of cholestatic jaundice in infants: recommendations of the North American Society for Pediatric Gastroenterology,
Hepatology and Nutrition. J Pediatr Gastroenterol Nutr 2004;39(2):115-28.

23. Suchy FJ. Neonatal cholestasis. Pediatr Rev 2004;25(11):388-96

24. Jacquemin E, Lykavieris P, Chaoui N, Hadchouel M, Bernard O. Transient neonatal cholestasis: origin and outcome. J Pediatr 1998;133(4):563-7.

25. Méndez V, Burastero M, Cuadro MN, Casamayou L, GutiéRrez C, Montano A, et al. Colestasis neonatal: evolución de los pacientes diagnosticados entre 1983 y 2000 en dos servicios gastroenterológicos de referencia de Montevideo. Arch Pediat Urug 2005;76:210-21.

26. Mowat AP, Davidson LL, Dick MC. Earlier identification of biliary atresia and hepatobiliary disease: selective screening in the third week of life. Arch Dis Child 1995;72(1):90-2.

27. Roberts EA. Neonatal hepatitis syndrome. Semin Neonatol 2003;8(5):357-74

28. Vidal Duran C, Ureche Aguarán G. Hepatitis A colestásica. A propósito de un caso. Rev Col Gastroenterol 2006;21:225-9.

29. Glassman MS, Dellalzedah S, Beneck D, Seashore JH. Coincidence of congenital toxoplasmosis and biliary atresia in an infant. J Pediatr Gastroenterol Nutr 1991;13(3):298-300.

30. Robino L, Machado K, Montano A. Neonatal cholestasis due to congenital toxoplasmosis: Case report. Arch Argent Pediatr 2013;111(4):e105-8.

31. Tavares LA, Bittar D, Teixeira S. Relato de caso de colestase neonatal ocasionada por Toxoplasma gondii. HU Revista, Juiz de Fora 2009;35(4):324-7.

32. Donat Aliaga E, Polo Miquel B, Vila Carbó JJ, Sanguesa Nebot C, García-Sala Viguer C, Hernández Martí M, et al. Atresia de vías biliares: estudio clínico retrospectivo. An Pediatr (Barc) 2004;79(02):323-9.

33. Sepulveda Hincapie ME, Yepes Palacio NL, Gutierrez Mendoza FA. Hepatopatía crónica en niños. Justificación para un programa de transplante hepático infantil. Iatreia 2002;15(1):35-42.

34. Rosenthal P, Podesta L, Sher L, Makowka L. Liver transplantation in children. Am J Gastroenterol 1994;89(4):480-92. 